\title{
ON THE AUTOMORPHISM GROUP OF A LIE GROUP
}

\author{
D. WIGNER
}

ABSTRACT. It is proved that the automorphism group of a connected real or complex Lie group contains an open real or complex algebraic subgroup. It follows that the identity component of the group of complex automorphisms of a connected complex Lie group is a complex algebraic group.

Let $G$ be a connected real or complex Lie group, $\widetilde{G}$ its universal cover. It is well known that the group of real or complex automorphisms of $\widetilde{G}, A(\widetilde{G})$, is isomorphic to the algebraic group of real or complex automorphisms of the Lie algebra of $G$ (cf. [2, p. 97]). If $D$ is the kernel of the natural projection from $\widetilde{G}$ to $G$, the automorphism group of $G, A(G)$, is isomorphic to the subgroup of $A(\widetilde{G})$ which stabilizes $D$. The topology inherited by $A(G)$ as a subgroup of the general linear group of the Lie algebra of $G$ coincides with the topology of uniform convergence on compact sets of $G$. We will prove the following theorem, which is true when the base field is the real or complex numbers.

Theorem 1. The group of automorpbisms of the Lie group G contains an open algebraic subgroup.

I would like to thank G. P. Hochschild and G. D. Mostow for suggesting this problem and for many helpful discussions. In particular it was Professor Hochschild who first observed that the method of proof could be extended to the case where the base field is the field of real numbers.

We denote the radical of the commutator subgroup $[\tilde{G}, \tilde{G}]$ of $\tilde{G}$ by $N$.

Lemma 1. The inner automorphisms effected by elements of the unipotent radical $N$ of $[\widetilde{G}, \widetilde{G}]$ form a normal unipotent algebraic subgroup $V$ of $A(\tilde{G})$.

Proof. $V$ is normal since $N$ is stable under the action of $A(\widetilde{G})$ on $\widetilde{G}$. By [2, Chapter XI, Theorem 3.2] $V$ is unipotent, and $V$ is connected since it is the continuous image of the connected group $N$. By $[3, \S 10$, The orem 10.1] $V$ is algebraic.

Received by the editors June 5, 1973.

AMS (MOS) subject classifications (1970). Primary 22E10, 22E 15. 
Proposition 1. If $\tilde{G}$ is solvable and $d$ is in the center of $G$, then the subgroup $U$ of $A(\widetilde{G})$ consisting of the elements fixing $d$ is an algebraic subgroup.

Proof. Here the group $N$ of Lemma 1 coincides with $[\widetilde{G}, \tilde{G}]$. If $d$ is in $N$, there is a unique element $v$ of the Lie algebra of $N$ whose exponential is $d$ since $N$ is simply connected and nilpotent. An element of $A(\tilde{G})$ fixes $d$ if and only if its differential fixes $v$. Thus, $U$ is algebraic in this case. If $d \notin N$ there is nevertheless some closed real one parameter subgroup $P$ of $\widetilde{G}$ containing $d$, by $[2$, Chapter 16 , Theorem 1.2]. Since $\widetilde{G}$ (viewed as a real analytic group) is simply connected and solvable, $P$ is a one-dimensional real vector group. If $E$ is the subgroup of $\widetilde{G}$ generated by $d$, then $E$ is therefore discrete, and $P / E=K$ is a maximal compact subgroup of $\tilde{G} / E$. Any other maximal compact subgroup $L$ of $\tilde{G} / E$ is conjugate to $K$, so we have $x \in \tilde{G} / E$ with $x L x^{-1}=K$. Also $K N=L N$ since both are the inverse image of the unique maximal compact subgroup of the abelian group $\tilde{G} / E N$. It is then clear that $x$ can be chosen from $N E / E$. It then follows that if $T$ is the element-wise fixer of $P$ in $A(\tilde{G})$, then $U=T V$.

Now $T$ and $V$ are algebraic, and $V$ is normal in $A(\tilde{G})$. In the complex case, this implies immediately that $U$ is algebraic (cf. [3, Corollary 5.4], noting that, since $V$ is normal and connected, the hypothesis that $T$ be connected is not needed for the result). In the real case, we note first that the factor groups $A(\widetilde{G}) / V$ and $T /(T \cap V)$ inherit affine algebraic group structures from those of $A(\widetilde{G})$ and $T$, respectively (having the appropriate universal mapping property for morphisms of affine algebraic groups), owing to the fact that $V$ and $T \cap V$ are unipotent affine algebraic groups (cf. [3, Theorems 10.3 and 14.2]). The injection $T \rightarrow A(\widetilde{G})$ induces, in the natural way, an injective morphism of affine algebraic groups $T /(T \cap V) \rightarrow A(\tilde{G}) / V$. By a result of Bialynicki-Birula and Rosenlicht [1], the image of an injective morphism of real algebraic groups is algebraic. Thus, the image of $T /(T \cap V)$ is an algebraic subgroup $K$ of $A(\tilde{G}) / V$. The inverse image of $K$ in $A(\tilde{G})$, with respect to the canonical morphism $A(\tilde{G}) \rightarrow A(\tilde{G}) / V$, is therefore an algebraic subgroup of $A(\tilde{G})$. Clearly, this inverse image is precisely $U$, so that $U$ is algebraic.

Proof of Theorem 1. Let $R$ be the radical of $\widetilde{G}$, and let $S$ be a maximal semisimple analytic subgroup of $\widetilde{G}$. Let $d$ be a central element of $\widetilde{G}$. We had $d=r s$, where $r$ is in $R$, and $s$ is in $S$, and $r$ and $s$ are uniquely determined 
by $d$. Observe that $s$ actually lies in the center of $S$ and that $r$ lies in the centralizer, $M$ say, of $s$ in $R$. As before, let $N$ denote the radical of $[\widetilde{G}, \widetilde{G}]$. If $u$ is any element of $R$ there is an element $v$ in $N$ such that $v^{-1} u$ normalizes $S[2, \mathrm{p} .129]$. From this and the fact that $d$ is central it follows that $v^{-1} u$ belongs to $M$. Hence we have $R=N M$. Now we see that $R / N$ is isomorphic (as an analytic group) with $M /(M \cap N)$ by the closed graph theorem (cf. Pontryagin [4]). The group $M \cap N$ is the group of all elements $v$ of $N$ such that $s v s^{-1}=v$. Since $N$ is simply connected and nilpotent, the exponential map for $N$ is bijective, whence we see that $M \cap N$ is connected. The factor group $M /(M \cap N)$ is connected, because it is isomorphic with $R / N$. Therefore, $M$ is connected. Thus, $M$ is an analytic subgroup of the simply connected solvable group $R$ which implies that $M$ is simply connected.

Now observe that $r$ lies in the center of $M$. Indeed, if $u$ is any element of $M$, we have $u r s=r s u=r u s$, so that $u r=r u$. Let $T$ denote the fixer of $r$ in $A(M)$. We know from Proposition 1 that $T$ is an algebraic subgroup of $A(M)$. Let $K(S)$ and $K(M)$ denote the stabilizers in $A(\tilde{G})$ of $S$ and $M$, respectively. Clearly, each of these is an algebraic subgroup of $A(\tilde{G})$. Hence the same is true for $K(S) \cap K(M)=J$, say. The restriction map $J \rightarrow$ $A(M)$ is evidently a morphism of affine algebraic groups. Hence the inverse image, $E$ say, of $T$ in $J$ is an algebraic subgroup of $J$, and hence also an algebraic subgroup of $A(\tilde{G})$.

Now let $F$ denote the fixer of $d$ in $K(S)$. It is easy to see that the elements of $F$ are precisely those elements of $K(S)$ which fix both $r$ and $s$. The automorphisms that fix $s$ evidently belong to $K(M)$. Hence we have $F \subset J$, whence $F=K(S) \cap E$. Therefore, $F$ is an algebraic subgroup of $A(\tilde{G})$. We have $A(\tilde{G})=V K(S)$, where $V$ is the group of inner automorphism effected by the elements of $N$. Hence the fixer of $d$ in $A(\tilde{G})$ is $V F$. Exactly as in the above proof of Proposition 1, we see that $V F$ is an algebraic subgroup of $A(\tilde{G})$. Thus, the fixer of $d$ in $A(\widetilde{G})$ is an algebraic subgroup of $A(\tilde{G})$.

Now let $D$ denote the kernel of the universal covering $\tilde{G} \rightarrow G$. It is clear from the last result above that the element wise fixer of $D$ in $A(\widetilde{G})$ is an algebraic subgroup of $A(\tilde{G})$. Clearly, this fixer contains the identity component of $A(G)$ since that identity component must act trivially on $D$. Therefore the element wise fixer of $D$ is open in $A(G)$. This completes the proof of Theorem 1. In the complex case, the identity component of $A(G)$ is even algebraic, since the topological identity component of any complex algebraic group is algebraic. 


\section{REFERENCES}

1. A. Bialynicki-Birula and M. Rosenlicht, Injective morphisms of real algebraic varieties, Proc. Amer. Math. Soc. 13 (1962), 200-203. MR 25 \#3936.

2. G. Hochschild, The structure of Lie groups, Holden-Day, San Francisco, Calif., 1965. MR $34 \# 7696$.

3. - Introduction to affine algebraic groups, Holden-Day, San Francisco, Calif., 1971. MR 43 \#3268.

4. L. Pontrjagin, Topological groups, GITTL, Moscow, 1938; English transl., Princeton Math. Series, vol. 2, Princeton Univ. Press, Princeton, N. J., 1939. MR 1,44 .

DEPARTMENT OF MATHEMATICS, UNIVERSITY OF MICHIGAN, ANN ARBOR, MICHIGAN 48104 